\title{
Review: aerobic exercise reduces systolic and diastolic blood pressure in adults
}

Whelton SP, Chin A, Xin X, He J. Effect of aerobic exercise on blood pressure: a meta-analysis of randomized, controlled trials. Ann Intern Med 2002 Apr 2;136:493-503.

\section{QUESTION: In people $\geq 18$ years of age, are interventions that include aerobic exercise more effective than interventions without aerobic exercise for reducing blood pressure?}

\section{Data sources}

Studies were identified by searching Medline (1966 to September 2001) and SPORTDiscus and by reviewing bibliographies of relevant articles.

\section{Study selection}

Studies published in English were included if they were randomised controlled trials (RCTs) comparing interventions that included aerobic exercise (treatment group) with interventions without aerobic exercise (control group), lasted $\geq 2$ weeks, reported changes in blood pressure (systolic, diastolic, or both) from baseline to follow up with the corresponding variances or data to estimate them, and participants were $\geq 18$ years of age. All frequencies, intensities, and types of aerobic exercise were considered.

\section{Data extraction}

Three reviewers independently extracted data on sample size, participant characteristics, study design, details of the intervention, study duration, and outcomes. Outcomes included changes in blood pressure (systolic, diastolic, or both) from baseline to follow-up.

National Heart, Lung and Blood Institute and National Institutes of Health.

For correspondence:

DrJ He, Tulane

University School of

Public Health and

Tropical Medicine, New

Orleans, LA, USA.

Emailjhe@tulane.edu. and subgroup analysis showed that reduction in systolic and diastolic blood pressure was greater in the treatment group than in the control group.

\section{Conclusion}

In adults, aerobic exercise is effective for lowering systolic and diastolic blood pressure.

\section{COMMENTARY}

The recently published paper by Whelton $e t$ al is the largest meta-analysis to measure the effect of aerobic exercise on blood pressure to date. Previous meta-analyses have shown more dramatic effects of exercise on blood pressure, which may be attributed to trials of shorter duration with better patient compliance or exercise supervision. The metaanalysis by Whelton $e$ al shows that similar blood pressure reductions were achieved with low, moderate, and high intensity exercise and that little benefit was gained from exercising for more than 2.5 hours/week. Physicians who recommend even modest increases in physical activity are therefore providing valuable advice.

Both short and long term increases in physical activity have been achieved by using physician-based exercise interventions. ${ }^{12}$ Increasing physical activity or physical fitness is popular with patients and physicians ${ }^{3}$ and has an effect on blood pressure similar to that of other non-pharmacological interventions. Interventions for weight loss (eg, with a weight loss of about $4.5 \mathrm{~kg}$ ), reduction of dietary sodium, ${ }^{4}$ or lowering alcohol intake all have the potential of reducing blood pressure by 1 to $4 \mathrm{~mm} \mathrm{Hg}$ for at least 6 months.

Aerobic exercise of even modest duration, frequency, and intensity is a useful adjunct to other lifestyle changes and antihypertensive medications in the treatment of essential hypertension. It should be recommended to all patients with hypertension who are willing and able to try it.

C. Raina Elley, MBChB Bruce Arroll, MBChB PhD University of Auckland Auckland, New Zealand

1 Calfas KJ, Long BJ, Sallis JF, et al. A controlled trial of physician counseling to promote the adoption of physical activity. Prev Med 1996;25:225-33.

2 The writing group for the Activity Counseling Trial Research Group. Effects of physical activity counseling in primary care: the Activity Counseling Trial: a randomized controlled trial. JAMA 2001;286:677-87

3 Arroll B, Jenkins S, North D. Non-pharmacological management of hypertension: results from interviews with 100 general practitioners. J Hypertens 1996;14:773-7.

4 Effects of weight loss and sodium reduction intervention on blood pressure and hypertension incidence in overweight people with high-normal blood pressure. The trials of hypertension prevention, phase II. The trials of hypertension prevention collaborative research group. Arch Intern Med 1997;157:657-67. 